\title{
Three-dimensional anatomy of the transantral intraseptal infraorbital canal with the use of cone-beam computed tomography
}

L. Cârstocea, M.C. Rusu, C. Pascale, M. Săndulescu

"Carol Davila" University of Medicine and Pharmacy of Bucharest, Romania

[Received: 20 August 2019; Accepted: 13 September 2019]

The transantral or ectopic infraorbital canal (IOC) courses diagonally through the maxillary sinus (MS), thereby being exposed to risk during a number of surgical procedures. A few prior reports have presented evidence of a septa-embedded IOC, albeit only on single-plane slices. We identified this extremely rare variation of the IOC during a retrospective study of the cone-beam computed tomography files of 2 patients. In the first case, which involved a 34-year-old female patient, the canals and septa within the MS were bilaterally asymmetrical. On the right side, the sinus roof was attached to a short transverse septum that was traversed by the IOC, while the left sinus featured an oblique large septum that divided it into antero-superior and posterior chambers. The left IOC was embedded within the septum rather than within the orbital floor above the septum. In the second case, which concerned a 36-year-old male patient, the left MS featured an almost completely oblique/vertical septum that divided it into anterior and posterior chambers and also embedded the respective IOC, which was thus absent from the orbital floor. In both cases, infraorbital recesses in the anterior chambers of the MS were found that, if not documented on three-dimensional (3D) renderisations, could have been misidentified as infraorbital (Haller) cells. To the best of our knowledge, this is the first report to document the 3D anatomy of an extremely rare variant, namely a septum-embedded transantral IOC. Such a variant, if not adequately documented preoperatively, could divert the transmaxillary corridors down false paths or else expose the IOC to damage during surgical procedures involving access to tumours. (Folia Morphol 2020; 79, 3: 649-653)

Key words: maxillary nerve, infraorbital nerve, antrum of Highmore, canalis sinuosus, cone-beam computed tomography

\section{INTRODUCTION}

The infraorbital nerve (ION) is a continuation of the maxillary nerve and it courses through the roof of the maxillary sinus (MS), first in the infraorbital groove (IOG) and then in the infraorbital canal (IOC), before exiting anteriorly through the infraorbital foramen (IOF).
A number of prior studies have attempted to pattern the variable morphology and course of the IOC. For instance, Acar et al. [1] recently proposed a four-point classification system in which the first two types indicate an intramural course on the part of the $\mathrm{IOC}$ within the antral roof, while type 3 indicates

Address for correspondence: Dr. M.C. Rusu, "Carol Davila" University of Medicine and Pharmacy, 8 Eloilor Sanitari Blvd., RO-050474 Bucharest, Romania, e-mail: anatomon@gmail.com 
a transantral course and type 4 indicates a lateroantral course. However, although the transantral IOC has previously been included in a classification system, the transantral intraseptal IOC remains a rarely encountered variant, which has not previously been fully documented spatially. An oblique, IOC-containing septum could easily be confused with a normal IOC-containing orbital floor, which would divert the transmaxillary corridor to an incorrect, if not dangerous, path.

To the best of our knowledge, no previous reports have demonstrated the transantral course of an IOC embedded within a septum that consistently divides the MS. We hereby report on such an unusual anatomical variation, which is, for the first time, three-dimensionally documented in the present study.

\section{CASE REPORTS}

\section{Case 1}

The archived cone-beam computed tomography (CBCT) files of a 34-year-old female patient were retrospectively studied. The patient had been scanned for dental medical purpose using an iCat CBCT machine (Imaging Sciences International, Hatfield, PA, USA) with the following settings: a resolution of 0.250 , a field of view of $130^{\circ}$ and an image matrix size of $640 \times 640$. During the scan, the patient was positioned according to the manufacturer's instructions. The CBCT data were analysed using the iCatVision software and a related application, three-dimensional (3D) volume renderisations (3DVR) v5.0.0.3. The data were then exported as a single uncompressed DICOM file, which was additionally analysed using the Planmeca Romexis Viewer 3.5.0.R software, an approach that has been applied in other studies $[9,10]$. We evaluated two-dimensional (2D) multiplanar reconstructions (MPRs) in all three anatomical planes, as well as 3DVR of specific areas. The relevant anatomical features were exported as image files ( ${ }^{*}$.tif). The patient had provided written informed consent for all her medical data (including radiographs, CBCT scans and intraoral images) to be used for research and teaching purposes, provided that her anonymity and confidentiality were maintained.

The patient's IOCs were found to be bilaterally asymmetrical. Morphologically speaking, the right IOC followed an initial course within the MS roof (orbital floor), before descending in a transantral course towards the IOF (Fig. 1A, B). The left IOC followed a completely transantral course. The angle between the transantral IOC and the MS roof was $17.2^{\circ}$ on the right side and $23.1^{\circ}$ on the left side. The lengths of the right and left transantral segments of the $\mathrm{IOC}$ were $14.15 \mathrm{~mm}$ and $17.55 \mathrm{~mm}$, respectively. The right IOF was located $9.91 \mathrm{~mm}$ inferior to the infraorbital margin. On the left side, that distance was found to be $12.01 \mathrm{~mm}$.

When a series of coronal slices was evaluated, the air spaces within the MS (infraorbital recesses of the MS) appeared as false infraorbital ethmoid cells (Haller cells), which were not draining into the lateral nasal wall (Fig. 1C-E). On the coronal slices, it was possible to identify the short transverse septum of the right MS roof, which was also observed on the sagittal slices. The left MS contained a morphologically complex septum, which was further evaluated by means of 3DVR.

The 3D anatomical evaluation of the intrasinus morphology (Fig. 1F, G) demonstrated that the antero-supero-medial angles of the MSs did indeed contain infraorbital recesses rather than Haller cells. The short transverse septum of the right sinus roof appeared as an anatomical demarcation between the anterior and posterior parts of the sinus. On the left side, a large oblique intrasinus septum separated the antero-superior and postero-inferior chambers of the sinus, and it also embedded the IOC. The air spaces observed on the 2D coronal slices were found to be parts of the antero-superior chamber. The transverse facial part of the canalis sinuosus also appeared to be morphologically asymmetrical. More specifically, on the right side, the canal was embedded within the anterior antral wall, while on the left side, it was attached to the respective antral wall by a small ridge or buttress.

\section{Case 2}

The CBCT files of a 36-year-old male patient, who had been scanned for dental medical purpose using the same procedure and apparatus as described in relation to the first patient, were retrospectively studied.

On the left side, a pattern comparable to the variant reported on the left side in Case 1 was identified. In this second case (Fig. 2), the left MS exhibited an oblique-vertical frontal septum, which was incomplete medially and which almost completely divided the MS into two chambers. The anterior chamber was narrower, while the posterior chamber was larger. The $\mathrm{IOC}$ was located within the identified septum, which implies that it followed a transantral course. Hence, while on the right side the IOC was located within the orbital floor, on the left side it was located beneath the orbital floor. The length of the left transantral segment of the IOC was $14.88 \mathrm{~mm}$. 

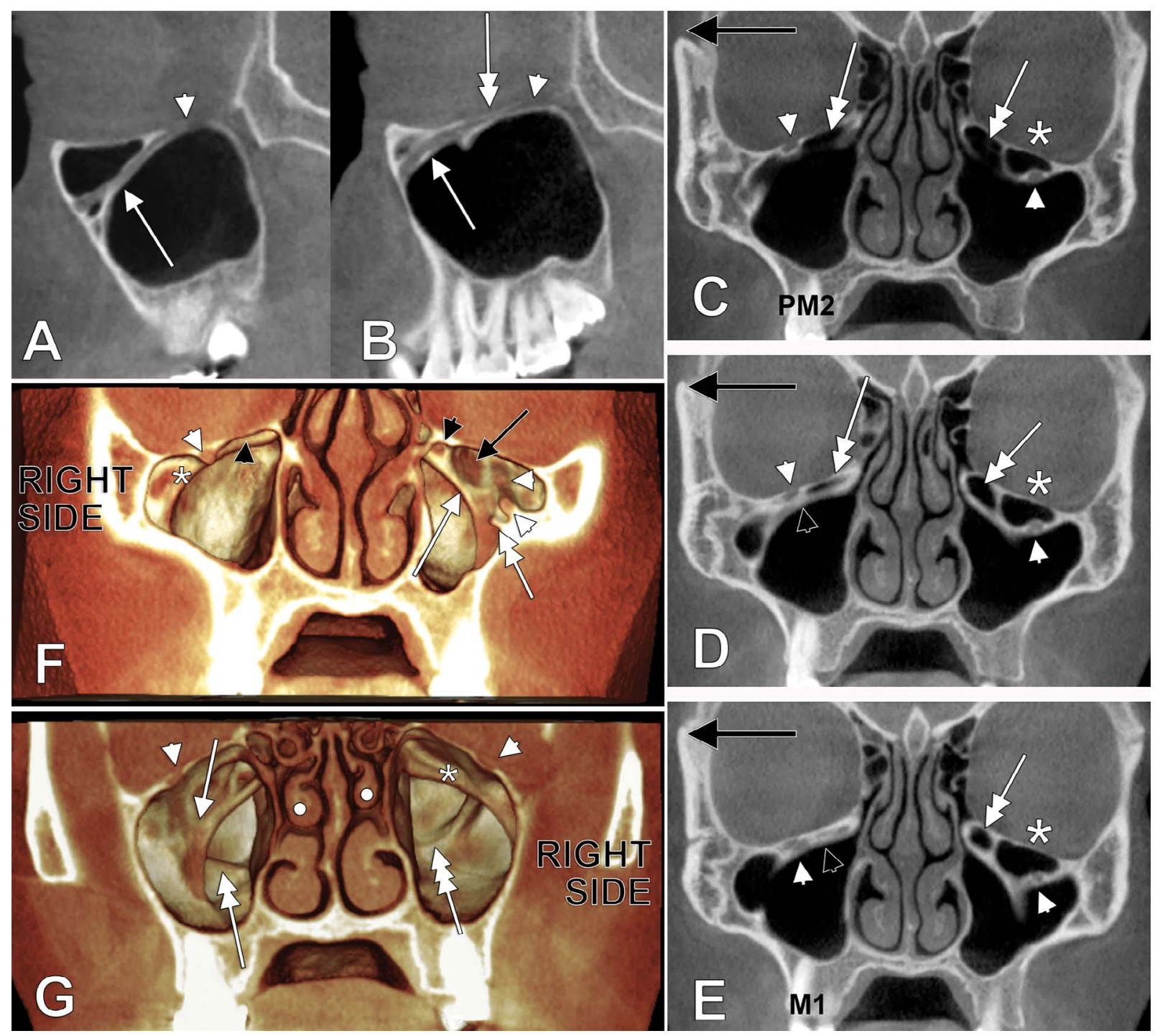

Figure 1. A, B. Sagittal slices through the maxillary sinuses. On the left side (A) the infraorbital groove (arrowhead) continues directly with a transantral infraorbital canal (arrow). On the right side (B) the infraorbital groove (arrowhead) continues with a roofed initial segment of the infraorbital canal located in the antral roof (double-headed arrow) further continued with a transantral segment (arrow); C-E. Antero-posterior series of coronal slices at the level of the upper second premolar (PM2) and first molar (M1). The black arrows indicate the right side. The infraorbital canals are indicated (white arrowheads). Bilaterally, infraorbital recesses of the maxillary sinuses (A, double-headed arrows) appear on successive slices as false Haller cells (B, C, double-headed arrows). The right infraorbital canal courses through a transverse septum of the respective sinus (B, C, black arrowhead). A distinctive recess is observed in the left maxillary sinus above the infraorbital canal $\left({ }^{*}\right)$; F, G. Three-dimensional volume renderisations of the maxillary sinuses, viewed anteriorly (F) and posteriorly (G). The infraorbital canals are indicated bilaterally (white arrowheads). On the right side a short transverse septum $\left(^{*}\right.$ ) attaches to the sinus roof to incompletely separate anterior and posterior chambers of the sinus. On the left side a large intrasinus septum (white arrow) attaches posteriorly to the sinus roof and is inclined antero-infero-laterally to separate antero-superior and postero-inferior sinus chambers. On the right side the sinuous canal courses within the anterior antral wall (B, triple-headed arrow). The left sinuous canal lies within the free border of a small ridge attached to the anterior antral wall (A, B, double-headed arrow). Infraorbital antral recesses are found bilaterally (A, black arrowheads). The air space above the left infraorbital canal ( $\mathbf{A}$, black arrow) belongs to the antero-superior chamber of that sinus. Bilateral paradoxical middle nasal turbinates were also found (B, white dots).

\section{DISCUSSION}

Accurate knowledge regarding the possible anatomical variations of the IOC is of paramount importance in relation to a number of maxillofacial procedures, including those intended to access MS tumours or orbital tumours and those intended to correct MS or orbital posttraumatic fractures [4]. The surgeon's field of view is narrowed during functional endoscopic sinus surgery, which, if it goes wrong, can lead to iatrogenic injuries to the IOC and subsequently to medico-legal issues [4].

Two different cases of an ectopic ION embedded within the bony septa crossing the upper part of the 


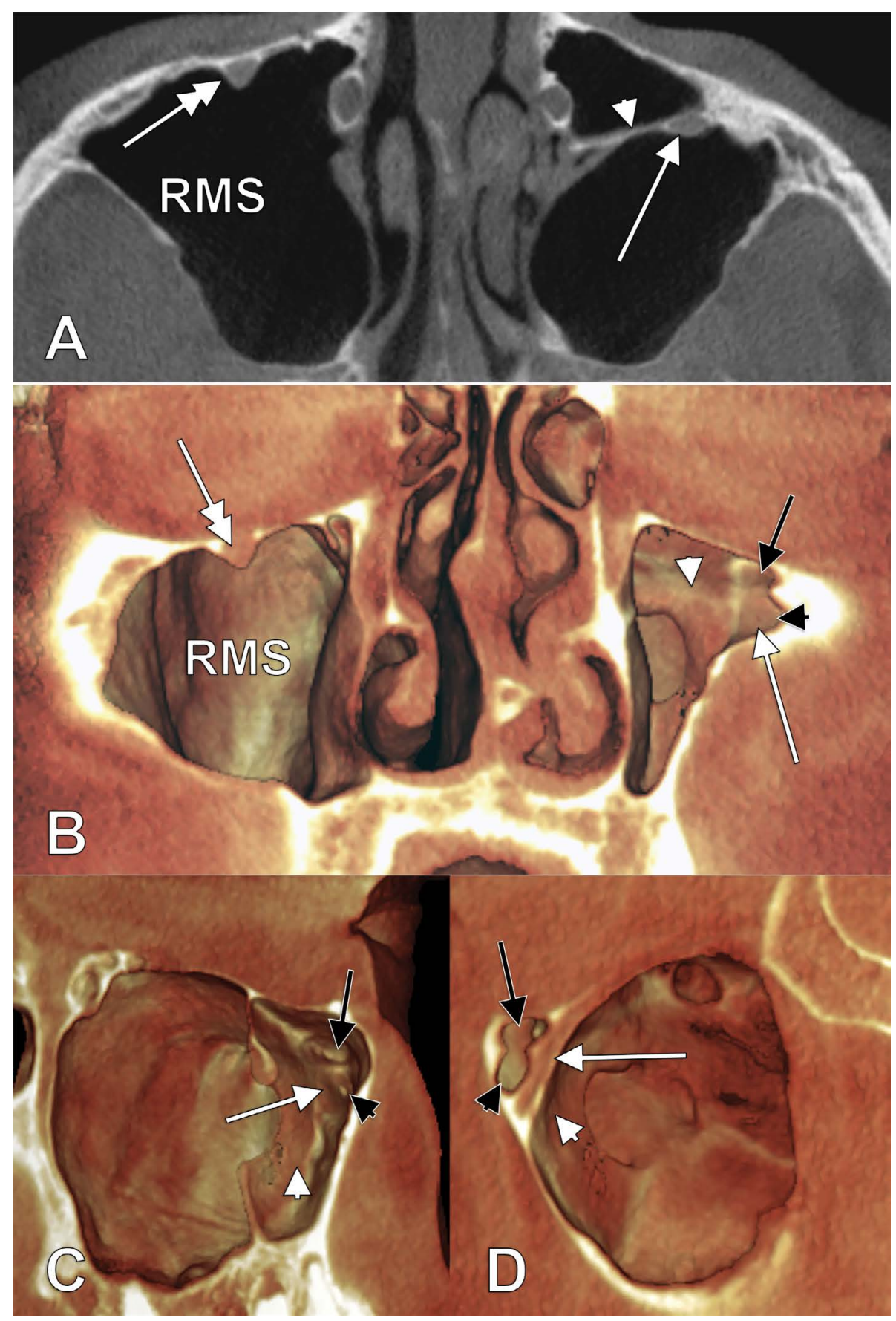

Figure 2. A. Axial slice through the maxillary sinuses; B. Three-dimensional volume renderisations of the maxillary sinuses, coronal cut, viewed anteriorly; RMS — right maxillary sinus; C. Three-dimensional volume renderisation of the left maxillary sinus, sagittal cut, viewed from lateral; D. Three-dimensional volume renderisation of the left maxillary sinus, sagittal cut, viewed from medial. There is indicated the right infraorbital canal (double-headed arrow) with a normal course. The left maxillary sinus is divided into anterior and posterior chambers by an almost complete coronal septum (white arrowhead) which embeds the left infraorbital canal (white arrow) which descends towards the infraorbital foramen. Above this canal and the respective septum two small infraorbital recesses are found, one superior (black arrow) and the other inferior (black arrowhead).

MS have been reported as very rare and previously unreported anatomical variations that could be dangerous during functional endoscopic sinus surgery [7]. Although the rarely occurring septum-embedded IOC has been demonstrated on coronal, sagittal and axial slices [7], the exact 3D architecture of such septa has not been presented, nor has the geometry of the IOC been detailed. The cases reported here showed that the IOC septa exhibited a transverse or oblique disposition, and that they partly separated the antero-su- 
perior or anterior and, respectively, postero-inferior or inferior MS chambers. Moreover, the authors [7] discussed how "septa in the maxillary sinus are present in about $16 \%$ of the maxillary septa... most often in atrophic maxillary sinuses (27\%)." The authors went on to quote a paper by Krennmair et al. (1997) [6], who clearly indicated that, when found, "all antral septa were located on the sinus floor". In these authors' study [7], the septa embedding the IOC occurred at a different anatomical situs than the sinus floor. However, this does not represent a rule, as it was systematically determined that $0.3 \%$ of the 1825 reported intrasinus septa divided the MS into two separate cavities [8].

More recently, another report concerning bilateral ectopic IONs has been published, albeit again without the inclusion of any 3D evidence [2]. Seemingly, in this recent report, the $\mathrm{IOC}$ septum was, as in the two previous reports, a transverse or oblique septum that only incompletely separated the two chambers of the MS.

In terms of the transantral type 3 IOC course, Ference et al. [3] determined the average diagonal length of the canal to be $15.40 \pm 3.06 \mathrm{~mm}$, which is in accordance with the present findings.

Frederic Wood Jones was the first to describe the canalis sinuosus, a neurovascular canal that conveys the superior anterior alveolar nerve and artery towards the premaxilla. As he wrote in 1939, "as the canal runs downwards in the anterior wall of the antrum, it frequently forms a prominent ridge or buttress on the inner aspect of the upper part of the antrum, between which and the buttress often formed by the infraorbital canal itself, there is commonly a deep recess." [5]. In the cases described in the present report, that ridge or buttress was unilateral, as on the opposite side the neurovascular content was embedded within the anterior antral wall.

\section{CONCLUSIONS}

In conclusion, although most prior studies have documented the sinus floor septa, the rarely encoun- tered septa attached to the sinus roof might have surgical relevance due to the fact that they relate to or embed the IOC.

\section{REFERENCES}

1. Açar G, Özen $K E$, Güler i, et al. Computed tomography evaluation of the morphometry and variations of the infraorbital canal relating to endoscopic surgery. Braz J Otorhinolaryngol. 2018; 84(6): 713-721, doi: 10.1016/j. bjorl.2017.08.009, indexed in Pubmed: 28943288.

2. Elnil H, Al-Tubaikh JA, El Beltagi AH. Into the septum I go, a case of bilateral ectopic infraorbital nerves: a not-to-miss preoperative sinonasal CT variant. Neuroradiol J. 2014; 27(2): 146-149, doi: 10.15274/NRJ-2014-10033, indexed in Pubmed: 24750699.

3. Ference EH, Smith SS, Conley D, et al. Surgical anatomy and variations of the infraorbital nerve. Laryngoscope. 2015; 125(6): 1296-1300, doi: 10.1002/lary.25089, indexed in Pubmed: 25992806.

4. Jakhere S, Kalmath V, Chillalshetti U. ctopic infra orbital nerves: case series of a dangerous normal variant. Acta Med Indones. 2018; 50(1): 66-69, indexed in Pubmed: 29686178

5. Jones FW. The anterior superior alveolar nerve and vessels. J Anat. 1939; 73(Pt 4): 583-591, indexed in Pubmed: 17104781.

6. Krennmair G, Ulm C, Lugmayr H. Maxillary sinus septa: incidence, morphology and clinical implications. J Craniomaxillofac Surg. 1997; 25(5): 261-265, doi: 10.1016/ s1010-5182(97)80063-7.

7. Mailleux $P$, Desgain $O$, Ingabire MI. Ectopic infraorbital nerve in a maxillary sinus septum: another potentially dangerous variant for sinus surgery. JBR-BTR. 2010; 93(6): 308-309, doi: 10.5334/jbr-btr.347, indexed in Pubmed: 21381529.

8. Pommer B, Ulm C, Lorenzoni M, et al. Prevalence, location and morphology of maxillary sinus septa: systematic review and meta-analysis. J Clin Periodontol. 2012; 39(8): 769-773, doi: 10.1111/j.1600-051X.2012.01897.x, indexed in Pubmed: 22624862.

9. Rusu MC, Sava CJ, llie AC, et al. Agger nasi cells versus lacrimal cells and uncinate bullae in cone-beam computed tomography. Ear Nose Throat J. 2019; 98(6): 334-339, doi: 10.1177/0145561319840836, indexed in Pubmed: 31012345

10. Sava CJ, Rusu MC, Săndulescu M, et al. Vertical and sagittal combinations of concha bullosa media and paradoxical middle turbinate. Surg Radiol Anat. 2018; 40(7): 847-853, doi: 10.1007/s00276-018-1998-0, indexed in Pubmed: 29502247. 